\title{
Research On Analogy Cellular Automata Model Inaggregation Of Operational Capability
}

\author{
Qiong Wang \\ Department of Information Engineering \\ Academy of Armored Force Engineering \\ Beijing, China \\ e-mail: holywang_zju@163.com
}

\author{
Xinhua $\mathrm{He}$ \\ Department of Information Engineering \\ Academy of Armored Force Engineering \\ Beijing, China \\ e-mail: hxh717495@tom.com
}

\author{
Qisheng Guo \\ Department of Equipment Command and Administration \\ Academy of Armored Force Engineering \\ Beijing, China
}

\begin{abstract}
Operational capability aggregation of armaments systems is significant for its future developing, planning, manufacturing and synthesis using. Holistic avalanche character of armaments systems can't be represented in traditional linear aggregation of operational capability, which regards the "system" or "platform" as the core. Cellular automata that indicate the holistic effect of system evolvement by processing partial conversions is firstly introduced on this paper, on the base of which, analogy cellular automata model is proposed, combing with applied details of operational capability aggregation. Experiment results prove that the model is valid.
\end{abstract}

Keywords-armaments systems; operational capability aggregation; holistic avalanche character; analogy cellular automata model

\section{INTRODUCTION}

The war in the future is the war information-based, the character of which is armaments systematic combat on the base of information system [1]. And the result of armaments systematic combat is enslaved to connatural operational capability of armaments system. The researches on operational capability aggregation of armaments system is significant for armaments systems' future developing, planning, manufacturing and synthesis using, regarded as a hot topic in martial fields.

The interplay among systems and system components enhances, along with advancing of armaments systems' information level. The traditional operational capability analyses which regards "system" or "platform" as the core, neglect the interaction and chain effect resulted by multi-net linkage and multi-field connection of systematic components, which not fit for operational aggregation of armaments systems. Holistic avalanche character [2] of systematic operational capability can't be represented with linear adding of multi "system" or "platform", and the aggregation can't reflect the nonlinear character of operational capability.

First, operational capability aggregation of armaments systems and its research status quo is introduced on this paper. And then, analogy cellular automata model in operational capability aggregation is proposed based on original cellular automata model, combing with specific applying of operational capability aggregation. Last, relative experiments are carried out, by which validity of the model is proved.

\section{OPERATIONAL CAPABILITY AGGREGATION OF ARMAMENTS SYSTEMS}

\section{A. Armaments System}

Armaments system is defined as: armaments system is higher system made up of various equip systems that interactional and interrelated in the condition of certain strategic guidance, combat command and support, in order to accomplish determinate assignments [3].

\section{B. Operational Capability Aggregation}

Operational capability aggregation of armaments system is denoted as "ability" or "potential" to carry out certain assignments, which is connatural character of armaments system, is up to its quality, quantity and apply manners in combat, and has nothing to do with specific apply process [4].

Operational capability aggregation is that new and enhancive operational capability is formed to meet the demands of combat applies by combing and integrating rock-bottom capability with determinate layer character. That is to say, operational capability aggregated is greater that the summation of sub-operational capabilities, which reflect the system's avalanche character.

\section{Analogy Cellular Automata Model}

\section{A. Cellular Automata}

Original cellular automata model $[5,6]$ is used for simulating the function of self-replicate in life system by Von Neumann. Cellular automata is a sort of kinetic system with discrete time, discrete space and discrete states, in which each discrete time step, every cell synchronously 
evolves with partial rules, according to its neighbors' states in last time step.

Generally speaking, cellular automata can be described in formal language as a quaternion: $A=(L, S, N, f)$, where $A$ denotes a cellular automata, $L$ denotes the set of cells in gridding space, $S$ denotes the set of cell states, $N$ denotes the set of neighbors and for each cell's neighbors that $N \subseteq L, f$ denotes state transform function of cells, as follows: $f: s_{t+1}^{i}=f\left(s_{t}^{i}+s_{t}^{n}\right)$ which indicates the state of cell $i$ in time step $t+1$ is up to its state and its $n$ neighbors' states in time step $t$ according to transform rules. The cells and their states, the cell space, the neighbors and partial transform rules are regarded as essential components of cellular automata.

Differ from other kinetic models, cellular automata does not has strict physical equation or function, but made up of a series of modeling rules. Any model satisfies the rules can be considered as cellular automata models. Hence, cellular automata model is just a name of a sort of models, or can be called as methodological frame. The main characters of cellular automata is that the time, space and states in cellular automata is discrete and each cell only has limited states, furthermore transform rules of cell states is partial in both time and space.

\section{B. Analogy Cellular Automata Model}

The system is regarded as one, and mapping relationship of system inputs and outputs is set up in traditional modeling. However, cellular automata pay attention to the interplay among partial elements and components, and form the holistic change of system by partial transform. Because of its simple, open and self-organizing characters of the frame, cellular automata model owns a great life-force in researches on complex system.

The discrete character of cellular automata brings good operability, however, applies in non-discrete system are limited in a certain extent at the same time. In order to meet the demand of actual apply, the set of cell states is extended to continuous space, and analogy cellular automata model is proposed on this paper.

Define: An analogy cellular automata model can be denoted by a quaternion as: $A^{+}=\left(L, S_{d}, N, f\right)$, where $L$ denotes the set of cells in space structure, $S_{d}$ denotes the continuous space made up of cell states, $N$ denotes the set of neighbors and for each cell's neighbors that $N \subseteq L, f$ denotes state transform function of cells, as follows: $f: s_{t+1}^{i}=f\left(s_{t}^{i}+s_{t}^{n}\right)$ which indicates the state of cell $i$ in time step $t+1$ is up to its state and its $n$ neighbors' states in time step $t$ according to transform rules. Details as follows:

1) $L$ denotes the set of cells in space structure. In order to adapt to the situation of non-regular gridding structure, the cells distributed in space structure are regarded as the main subjects of analogy cellular automata.

2) $S_{d}$ denotes the continuous space made up of cell states. When the set of cell states is limited and discrete in cellular automata, in analogy cellular automata it is infinite and continuous. $d$ denotes the dimension of the state space.

3) $N$ denotes the set of neighbors. The cell is considered as partial element of system, while its neighbors as other partial elements related to it. Here, neighbors are classified into different classes according to the way the interplay takes. Hence, $N$ can be denoted as $N=\sum N_{i}$, where $N_{i}$ denotes the set of neighbors in the same class.

4) $f$ denotes transform function of cell states. Cell states are affected by its self-state and its neighbors' states. Combining with classify of neighbors, the transform rules are denoted as:

$$
f: s_{t+1}=f\left(s_{t}+\sum_{i} s_{t}^{n_{i}}\right), \text { where, } s_{t}^{n_{i}} \text { denotes the set of class }
$$
of neighbors.

All in all, comparing with cellular automata, analogy cellular automata owns these characters as follows: 1) the space structure of cells is no longer restricted in regular gridding structure, but dispersive space structure; 2) neighbors are redefined and classified, and several simple relation model is used to define the transform rules; 3 ) the cell states are extended into infinite continuous space from original limited discrete set, so that the model can be used for some continuous system.

\section{Analogy Cellular Automata Model IN OPERATIONAL CAPABILITY AGGREGATION}

\section{A. The Cell Of Operational Capability}

Operational capability is the basic unit of operational capability aggregation of armaments system. In traditional aggregation model of operational capability, a tree-like structure with hiberarchy is made up of the whole operational capability. However, as enhancing in equip informationization, functional interdependence among systems and platforms also enhances, what it results is that the existing relation structure of operational capability can't be described accurately by traditional structure. Especially, as armaments system, the relationship among composing systems is both independence and interrelation. Operational capabilities as the functional mapping of armaments system, among which, the interplay is complicate, so that the complex space network structure is made up.

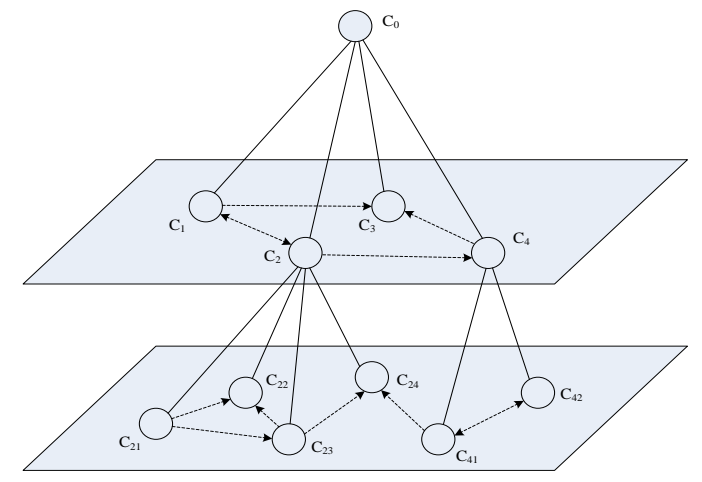

Figure 1. Space structure of operational capabilities (part) 
Fig. 1 shows part of space structure of armaments system's operational capability, the real line constructs the traditional tree-like structure of operational capability, which denotes aggregation relationship between upper and lower capabilities. The broken line constructs the space network structure, which denotes the interplay among capabilities.

The aggregation relationship between upper and lower capabilities can be divided into two sorts [7] :

1) The relation of "or", and denoted by weighted sum model. Lower capabilities are aggregated to upper capability with different weights, of which the function is denoted as follows:

$$
C_{t}=\alpha_{1} C_{t 1}+\alpha_{2} C_{t 2}+\cdots+\alpha_{n} C_{t n}
$$

Where $C_{t}$ denotes upper capability, $C_{t i}$ denotes lower capability, and $\alpha_{i}$ denotes the weight of lower capability.

2) The relation of "and", and denoted by weighted product model. For upper capability, every lower capability is indispensable, although the weight is different. Once any lower capability's numerical value is zero, it will results that the upper capability be zero too. The function is denoted as follows:

$$
C_{t}=C_{t 1}^{\alpha_{1}} \cdot C_{t 2}^{\alpha_{2}} \cdots C_{t n}^{\alpha_{n}}
$$

Where $C_{t}$ denotes upper capability, $C_{t i}$ denotes lower capability, and $\alpha_{i}$ denotes the weight of lower capability.

In our past works, the concept of feedback in cybernetics was introduced into operational capability aggregation, researches on the mechanism of feedback among capabilities had been carried out [8]. The relation of interplay among capabilities can be denoted as follows:

$$
C_{t+1}^{i}=\left\{\begin{array}{l}
C_{0}^{i}+\Delta d, C_{t}^{j} \geq \sigma \\
C_{0}^{i}-\Delta d, C_{t}^{j}<\sigma
\end{array}\right.
$$

Where $\Delta d=g\left(\left|C_{t}^{j}-\sigma\right|\right)$ denotes the quantity of feedback that represents how much the capability $C_{t}^{j}$ affects $C_{t+1}^{i}$, $C_{0}^{i}$ denotes the original value of $C^{i}, \sigma$ denotes limen of feedback.

When the single operational capability taken as individual cell, the whole capabilities together construct the space structure of cells, and the cell states, neighbors and states transform rules are described, so that analogy cellular automata of operational capability aggregation is modeled, which represents the holistic effect of systematic operational capability by partial evolution.

Defines: In analogy cellular automata model of operational capability aggregation, $C_{i}$ denotes the cell $i$, $L$ denotes the space made up of all the cells, then it indicates: $C_{i} \in L, \quad i=1,2, \cdots, n$ 。

\section{B. The Cell States}

In the analogy cellular automata defined, the cell state is the dot in continuous space, the dimension of the space is up to the dimension of cell states. Capability as an individual cell, its exterior meaning is the numerical value of operational capability. Moreover, being quantified, the numerical value of operational capability is in a certain range, e.g. the range should be $0 \sim 10$ while adopting ten-point system.

Defines: in analogy cellular automata model of operational capability aggregation, the numerical value of capability is taken as the cell state, $s_{t}^{i}$ denotes the state of the cell $i$ in time step $t$, and $S$ denotes the continuous space made up of all the cell states. If $S$ takes the range [0,10], then it indicates: $s_{t}^{i} \in[0,10]$ 。

\section{The Cell Neighbors}

As to individual cell of operational capability, its neighbors is the set of cells related. We use to denote certain sort of neighbor set, and according to different type of effects, neighbors can be divided into two groups, as follows:

1) I-type neighbors, formed by aggregation from upper and lower capabilities, denoted as $N_{1}$. As to the cell, it's effected by the aggregation from lower capabilities, and on the other hand, it is one of I-type neighbors of upper capability.

2) II-type neighbors, formed by feedback among capabilities, denoted as $N_{2}$. When the cell is fed back by other capabilities, the capabilities that do feedback make up the set of this type neighbors.

\section{States Transform Rules}

There are two factors for system evolution in analogy cellular automata as follows: 1) the cells with original states; 2) the transform rules of the cell states. First, we sort the cells into three classes:

1) The cell of bottom layer capability. It has original value of operational capability that is the cell with original state, only owns II-type neighbors. $s_{0}^{i}$ denotes the original value of the cell state of $C_{i}$.

2) The cell of middle layer capability. It hasn't original value of operational capability, but takes the value aggregated by lower capabilities as the basic value for state transform. These cells own both I-type and II-type neighbors.

3) The cell of target layer capability. It hasn't original value of operational capability, but obtains the value aggregated by lower capabilities directly. These cells do not be fed back by any other cells, and it only owns I-type neighbors.

The states transform rules of the three sorts of cells will be shown as follow:

As to the cell state of bottom layer capability $C_{i}$ in time step $t+1$ : 


$$
s_{t+1}^{i}=s_{0}^{i}+f_{2}\left(s_{t}^{n_{2}}\right)
$$

Where $s_{t}^{n_{2}}$ denotes II-type neighbor of $C_{i}$, $f_{2}\left(s_{t}^{n_{2}}\right)$ denotes the quantity of feedback by II-type neighbor. Combing with formula (3):

$$
s_{t+1}^{i}=s_{0}^{i}+\sum_{j}^{n_{2}} g\left(s_{t}^{j}-\sigma\right)
$$

As to the cell state of middle layer capability $C_{i}$ in time step $t+1$ :

$$
s_{t+1}^{i}=f_{1}\left(s_{t}^{n_{1}}\right)+f_{2}\left(s_{t}^{n_{2}}\right)
$$

Where $s_{t}^{n_{1}}$ denotes I-type neighbor of $C_{i}, s_{t}^{n_{2}}$ denotes II-type neighbor, $f_{1}\left(s_{t}^{n_{1}}\right)$ denotes aggregating calculation, $f_{2}\left(s_{t}^{n_{2}}\right)$ denotes the quantity of feedback by II-type neighbor. Combing with formula (1) (2) (3):

$$
s_{t+1}^{i}=\sum_{k}^{n_{1}} \alpha_{k} s_{t}^{k}+\sum_{j}^{n_{2}} g\left(s_{t}^{j}-\sigma\right)
$$

or

$$
s_{t+1}^{i}=\prod_{k}^{n_{1}}\left(s_{t}^{k}\right)^{\alpha_{k}}+\sum_{j}^{n_{2}} g\left(s_{t}^{j}-\sigma\right)
$$

As to the cell state of target layer capability $C_{i}$ in time step $t+1$ :

$s_{t+1}^{i}=f_{1}\left(s_{t}^{n_{1}}\right)$

Where $s_{t}^{n_{1}}$ denotes I-type neighbor of $C_{i}$, $f_{1}\left(s_{t}^{n_{1}}\right)$ denotes aggregating calculation. Combing with formula (1) (2) (3):

$$
s_{t+1}^{i}=\sum_{k}^{n_{1}} \alpha_{k} s_{t}^{k}
$$

or

$$
s_{t+1}^{i}=\prod_{k}^{n_{1}}\left(s_{t}^{k}\right)^{\alpha_{k}}
$$

\section{E. System Evolution}

Analogy cellular automata inherit the character of system evolution from original cellular automata, which form the holistic transformation by partial changes. Analogy cellular automata of operational capability aggregation represent the holistic effect of systematic operational capability by the interplay among capabilities. While the numerical change of the target cell being smaller than a particle, the system reaches a steady state and the evolution is terminated. Then the state value of the target cell is regarded as the output of the aggregation model. The process of system evolution is shown as follows:

Step.1 Decompose the operational capabilities of armaments system layer by layer as top-down, analyze the relationship of the capabilities, and then set up the space structure of operational capabilities.

Step.2 Set up analogy cellular automata model and set the cell space, neighbors and state transform rules.

Step.3 Initialize the numerical value of bottom layer cells, set the particle $\varepsilon$, set $t=0$ and start system evolution.

Step.4 At the time step $t$, calculate the state values of all the cells by turn.

Step.5 Judge whether the numerical change of the target cell is smaller than $\varepsilon$, then go to the next step, or go back to step 4.

Step.6 Terminate the evolution, and output the numerical value of the target cell's state.

\section{INSTANCE ANALYSIS}

The model of analogy cellular automata is set up with $\mathrm{VC}++6.0$ to do simulation, which is integrated into the platform of operational capability aggregation of armaments. Take certain digital army's armaments system as an instance, applying experiments of analogy cellular automata model are carried out, and the results are analyzed while the model validated.

Armaments system of the digital army is made up of weapon system, joint tactical communication system, intelligence and reconnaissance system, command and control system, electronic warfare system and synthetical support system. According to hiberarchy character of armaments system, the systematic operational capability is decomposed and a three-layer structure of operational capabilities is set up as shown in figure 2 .

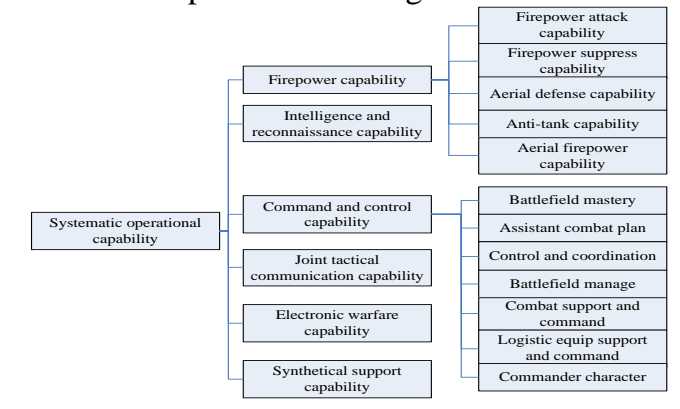

Figure 2. The index system of operational capabilities（part）

The relationship of operational capabilities is sorted into two groups according to applying manners and characters of armaments system as follows:

1) Relation of functional dependence. Certain operational capability depends on other capabilities with their systemic functions that form the sort of relationship. E.g. synthetical situation of the battlefield is offered for battlefield control capability by intelligence and reconnaissance capability; as 
well as command and control capability provide control function for recceing, and so on.

2) Relation of cooperation. Because of the cooperation in the same sort of capabilities, it brings the avalanche on holistic effects of the system. E.g. the holistic effect of joint fire attack brought by firepower attack capability, firepower suppress capability, aerial defense capability, anti-tank capability and aerial firepower capability, whose space structure of capabilities shown as figure 3 .

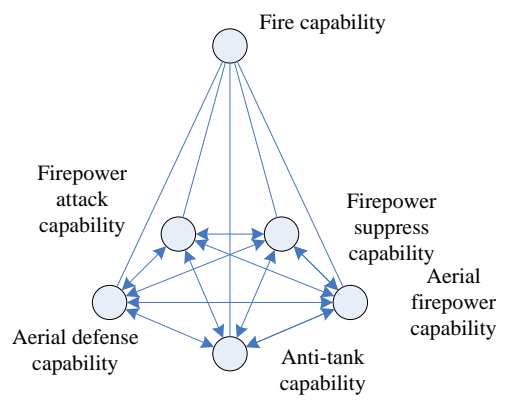

Figure 3. Space structure of fire capability

To be convenient for calculating, ratio model is adopted as the feedback calculation: $g\left(s_{t}-\sigma\right)=\lambda\left(s_{t}-\sigma\right)$ where $\lambda$ denotes the ratio, then $\lambda \in[0,1]$, and $\sigma$ denotes the limen of the feedback.

Simulating experiment about operational capability aggregation of the digital army is carried out, in which the bottom layer capabilities are quantified in ten-point system. There are several comparing groups as follows:

1) With the traditional aggregation which is linear.

2) With the model of analogy cellular automata, setting the particle $\varepsilon=0.0001$, the feedback limen $\sigma=6.0$, the feedback ratio $\lambda=0.1$.

3 ) With the model of analogy cellular automata, setting the particle $\varepsilon=0.0001$, the feedback limen $\sigma=6.0$, the feedback ratio $\lambda=0.12$.

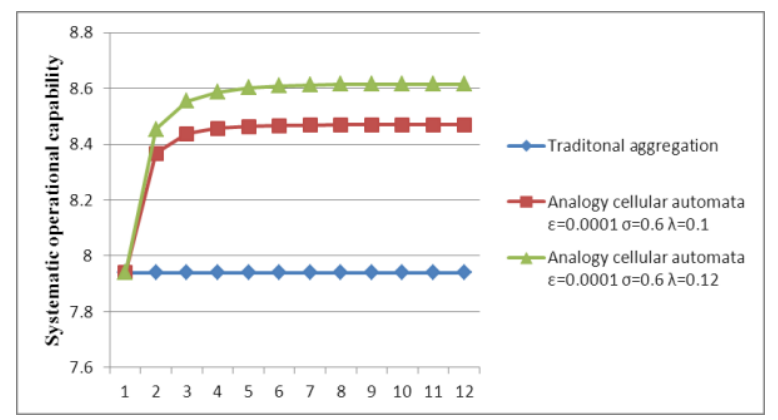

Figure 4. Experiment result I

Fig. 4 shows the result of comparing experiments. From the figure, it's found that systematic operational capability calculated by analogy cellular automata model is greater than the traditional aggregation's result, that's because analogy cellular automata model is set up based on the interplay among systems and system components, relation of functional dependence and congener capabilities' cooperation are considered synthetically, so that the aggregated result reflect the holist avalanche in a certain extent. The different results aggregated with different feedback quantities reflect how much the interplay effects systematic capability. Armaments system based on information system make use of information technology to realize the integration of all the weapon equip systems, of which the mechanism of information sharing and data exchange is the powerful multiplicator of armaments systematic operational capability, and the mature degree of information technology greatly affects the systematic capability.

On the other hand, another comparing experiment is carried out about the change of operational capability of armaments system before and after digitized. Table 1 shows part of bottom index values. The model of analogy cellular automata is used and set the particle $\varepsilon=0.0001$, feedback limen $\sigma=6.0$, feedback ratio $\lambda=0.12$. The result is shown as Fig. 5.

TABLE I. BOTTOM LAYER CAPABILITIES（PART）

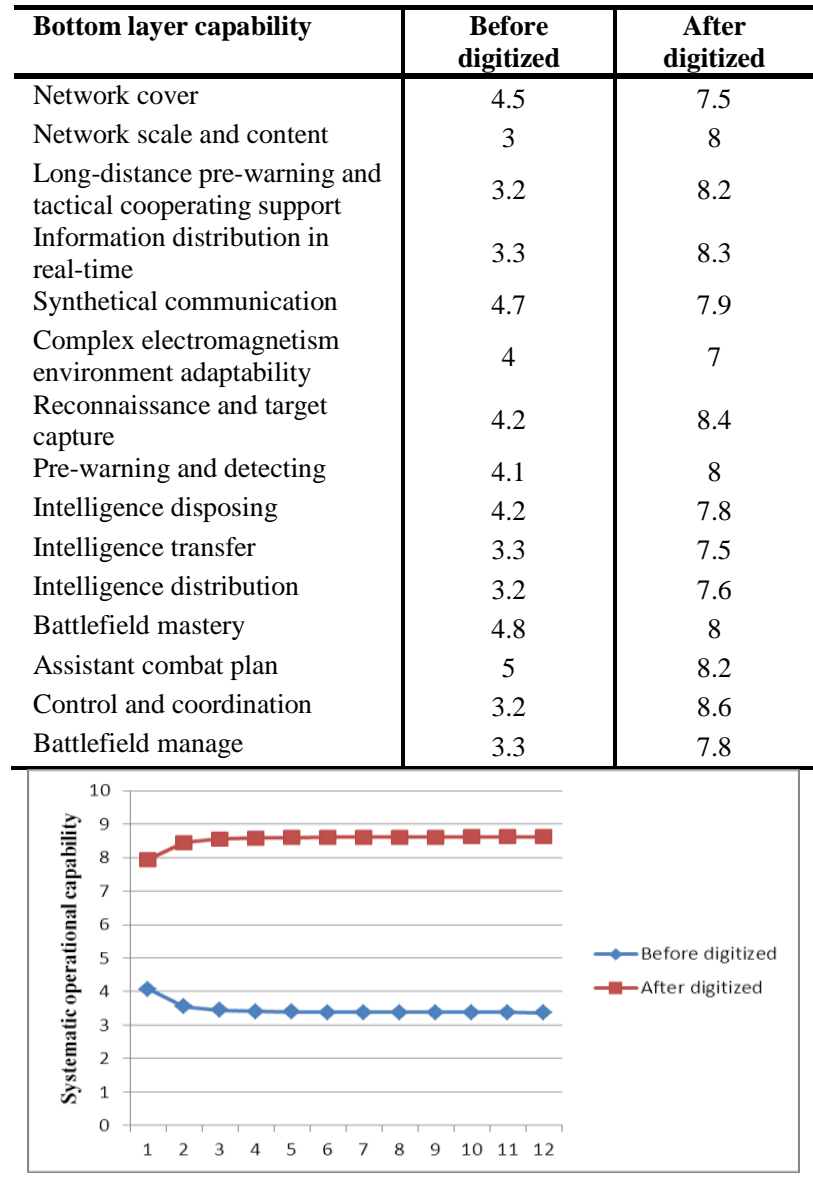

Figure 5. Experiment result II

From the table, it's found that the raise of weapon equip systems' operational capability after digitized not more than one time, but the systematic capability raise more than one time. That's because of information technology that realize 
great share of information, raise the systematic capability of armaments system greatly after digitized.

\section{CONCLUSION}

When the interplay among partial elements and system components is paid attention to, Cellular automata represent the holistic and macroscopical change of system by coupling partial changes. The model of analogy cellular automata in operational capability aggregation is proposed based on original cellular automata model, combing with specific applying of operational capability aggregation. The holistic effect of systematic operational capability is reflected by individual cell evolution. The instance analysis proves that the model is valid.

\section{REFERENCES}

[1] Hu Xiaofeng, YANG Jingyu, SI Guangya, et al. The war complex system simulation analysis and experimental. Beijing: National Defense University Press, 2008, pp.69-71.

[2] XU Guozhi, GU Jifa, CHE Hongan. Systems science. Shanghai: Shanghai education science and technology press, pp.200.

[3] NIU Xinguang. National defense system analysis of weapon equipment. Beijing: National Defense Industry Press, 2007, pp.32.

[4] LUO Peng-cheng, FU Pan-feng, ZHOU Jinglun. Framework to evaluate the combat capability of weapons SoS. Systems Engineering and Electronics, 2005, 27, No. 1, pp.72-75.

[5] Von Neumann. Theory of self-reproducing automata. Chicago: University of Illinois Press, 1996.

[6] Clarke K C, Gaydos L J, Hoppen S. A self-modifying celluar automation model of historical urbanization in the San Francisco Bay area. Environment and Planning B, 1997, No. 24, pp.247-261.

[7] HE Bo, LIU Xiao-dong, JIN Xiaochao. Research on aggregation model for air-force arming system combat capability. Fire Control \& Command Control, 2009, Vol. 34, No. 7, pp.92-94.

[8] HE Xinhua, WANG Qiong, GUO Qisheng, et all. Aggregation method of armament system operational capability based on feedback mechanism. Journal of Academy of Armored Force Engineering, 2012, Vol. 26, No. 5, pp.13-17. 